Ann. Biol. anim. Bioch. Biophys., I968, 8 (3), 373-383.

\title{
INFLUENCE DE LA DUREE DES PERIODES DE RATIONNEMENT APRÈS LE SEVRAGE SUR L'ÉVOLUTION DE LA COMPOSITION CORPORELLE DU RAT BLANC
}

\author{
B. DESMOULIN \\ avec la collaboration technique de M. Lecourtier et P. Peiniau \\ Station de Recherches sur l'Élevage des Porcs, \\ Centre national de Recherches zootechniques, 78 - Jouy-en-Josas \\ Institut national de la Recherche agronomique
}

\section{SOMMMAIRE}

L'expérience suivante a pour but de préciser l'influence du niveau énergétique d'alimentation et de sa durée d'application après le sevrage sur l'évolution ultérieure de la composition corporelle du Rat blanc : les animaux reçoivent une même quantité de protéines mais des quantités d'énergie différentes de 25 p. I00. Pour 2 lots : Haut-Haut (HH) et Bas-Bas (BB), les niveaux sont maintenus durant toute la période de croissance étudiée. Pour 4 lots, respectivement : Bas-Haut $(\mathrm{BH})$ et Haut-Bas (HB), les niveaux sont intervertis - soit 2 semaines - soit 4 semaines après le début de l'expérience.

Une restriction en énergie, imposée de façon continue depuis le sevrage, n'empêche pas une lipogenèse tardive chez le Rat soumis au rationnement Bas-Bas (BB). Cette évolution de la lipogenèse pourrait résulter de plusieurs processus. D'une part, la phase critique du sevrage s'accompagne d'une adaptation de l'animal au régime riche en glucides. Par ailleurs, une réduction de l'apport de nourriture entraîne une épargne des dépenses énergétiques. Chez l'animal restreint, cette épargne pourrait résulter de la rapidité de consommation des repas et ainsi du comportement alimentaire. L'adaptation des animaux à ce niveau Bas s'extériorise pleinement lorsque l'on applique consécutivement un niveau Haut. En rationnement Bas-Haut $(\mathrm{BH})$, la lipogenèse est d'autant plus stimulée que la durée de la restriction a été plus longue ; ceci dans les conditions d'une réalimentation contrôlée.

Un niveau énergétique élevé, appliqué de façon continu depuis le sevrage s'accompagne d'une lipogenèse précoce chez le rat soumis au rationnement Haut-Haut $(\mathrm{HH})$. Lorsqu'une restriction est imposée ultérieurement, le rat adapté au niveau Haut, accuse d'autant plus les effets de la restriction, que le niveau élevé d'alimentation a été maintenu plus longtemps après le sevrage. En rationnement Haut-Bas $(\mathrm{HB})$, la lipogenèse est limitée ou diminuée selon le stade de développement de l'animal au changement de niveaux. 


\section{INTRODUCTION}

Les conséquences d'une sous-alimentation sur le développement ultérieur de 1'animal sont controversées. Selon OSBORNE et MENDEL (I9I5), un arrêt de croissance imposé durant une longue période à des rats de $100 \mathrm{~g}$ ne modifie pas leur aptitude à reprendre leur poids avec un régime convenablement équilibré. Par contre, $\mathrm{MCCAY}$, MAYNARD et al. (I939) notent que la récupération de poids demeure incomplète lorsque la sous-nutrition a été imposée à des rats de $50 \mathrm{~g}$. Suivant le stade de développement auquel a été appliquée la restriction alimentaire, l'aptitude de l'animal à rétablir son niveau de croissance est donc différente (WILsoN-OSBOURN, I960). Cette aptitude est par ailleurs fonction de la nature de la restriction et de sa durée d'application. ClaRKe et Smrir (I948) ainsi que Widdowson (1964) montrent que le Rat blanc peut parfaitement surmonter ultérieurement l'effet de la carence énergétique lorsque sa durée d'application n'excède pas 6 semaines après le sevrage. D'autre part, les méthodes d'alimentation discontinue modifient l'intensité des reprises de poids lorsque celles-ci alternent avec des périodes de carences répétées (MORINJoMaIN, rg62-I963).

Des expériences antérieures (DESMOULIN, I967) ont montré que le Rat s'adapte au niveau de rationnement énergétique, Haut $(\mathrm{H})$ ou Bas $(\mathrm{B})$, qui leur est imposé lors du sevrage. Lorsque ces niveaux sont intervertis après 3 semaines de rationnement, l'efficacité des rétentions de l'énergie et de l'azote évolue différemment suivant le type de discontinuité : Bas-Haut (BH) ou Haut-Bas (HB). Dans les limites de poids considérées, les différences de composition corporelle sont conditionnées par la sévérité de la restriction imposée. On peut se demander si les résultats précédents sont également fonction de la durée des périodes de rationnement utilisées.

L'expérience suivante a pour objectif d'étudier l'évolution de la composition corporelle du Rat blanc lorsque l'interversion des niveaux d'alimentation Haut ou Bas depuis le sevrage, est effectuée après une période courte ( 2 semaines) ou longue (4 semaines). Dans les différents lots d'animaux soumis au rationnement continu ( $\mathrm{HH}$ et $\mathrm{BB}$ ) ou variable (BH et $\mathrm{HB}$ ), les compositions corporelles sont comparées à poids d'abattage identique.

\section{MATÉRIEL ET MÉTHODES}

\section{Animaux - Régimes et mode d'alimentation}

A la suite d'une période préexpérimentale d'une semaine après le sevrage, 8o Rats mâles de souche Wistar sont mis en expérience à l'âge de 4 semaines et au poids de $65 \pm 5 \mathrm{~g}$.

- Les animaux sont alors soumis à un rationnement journalier — soit à un niveau Haut qui est inférieur de Io p. Ioo au niveau spontané de la consommation du même régime - soit à un niveau Bas pour lequel les quantités distribuées sont inférieures de $25 \mathrm{p}$. 1oo à celles du rationnement Haut.

- Les régimes (I) riches en glucides (amidon et saccharose) renferment une farine de hareng de Norvège comme seule source azotée. Le régime qui est distribué au niveau Haut contient i $2 \mathrm{p}$. Ioo de protéines et celui distribué au niveau Bas, I6 p. Ioo de protéines $(\mathrm{N} \times 6,25)$. Compte tenu de la restriction alimentaire imposée, l'apport journalier de protéines est égalisé aux deux niveaux de rationnement. 
Régime Haut $(H)$ : Farine de poisson : 174,6; Amidon : 460,4; Saccharose : 215 ; Huile d'arachide : 80 ; Agar-agar : 20 ; Mélange salin : 40 ; Mélange vitaminique : Io.

Régime Bas $(B)$ : Farine de poisson : 232,8; Amidon : 402,2 ; Saccharose : 215 ; Huile d'arachide : 80 ; Agar-agar : 20 ; Mélange salin : 40 ; Mélange vitaminique : ro.

\section{Schéma expérimental - Mode d'abattage}

Durant deux périodes successives de leur croissance, les rats sont soumis suivant le schéma de MaC MeEkan (I940) aux différents types de rationnement par période : Haut-Haut (HH) Bas-Haut (BH) Haut-Bas (HB) et Bas-Bas (BB). L'inversion des niveaux est effectuée soit 2 semaines, soit 4 semaines après la période initiale de rationnement Haut $(\mathrm{H})$ ou Bas $(\mathrm{B})$ comme il est indiqué sur la figure I.

- L'état nutritionnel des animaux lors de la variation de niveau d'alimentation est précisé en effectuant des abattages de référence suivant l'âge : à 2 semaines pour $2 \operatorname{lots}\left(H_{2}\right.$ et $\left.B_{2}\right)$ à 4 semaines pour 2 lots $\left(H_{4}\right.$ et $\left.B_{4}\right)$.

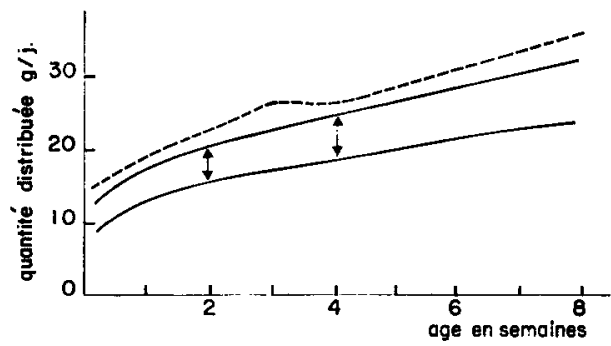

Fig. I. - Mode de rationnement selon l'áge

$\longleftrightarrow$ Changement de niveaux

$\longrightarrow$ échelle d'alimentation

-.n-n ad libitum

- Les abattages sont effectués ultérieurement au même poids final qui est celui atteint par les animaux du lot témoin (HH) à 6 semaines. Ces abattages concernent 6 lots :

- les 2 lots, $H H$ et $B B$, soumis au rationnement continu;

- les 4 lots, $H_{2} B$ et $B_{2} H, H_{4} B$ et $B_{4} H$, soumis au rationnement variable. Dans les lots $H B$ et $\mathrm{BH}$, l'indice affecté au rer traitement indique la durée (2 semaines ou 4 semaines) d'application du niveau d'alimentation correspondant.

\section{Mode d'expression des résultats - Composition corporelle}

L'influence du rationnement alimentaire sur la courbe de croissance est exprimée en rapportant la différence des vitesses de croissance entre la $2^{\mathbf{e}}$ et la $\mathrm{I}^{\mathrm{re}}$ période de rationnement à la vitesse de croissance observée au terme de la ${ }^{\text {re }}$ période. $\mathrm{GMQ}_{1}$ étant la vitesse de croissance durant la $I^{\text {re }}$ période et $G M Q_{2}$, celle durant la $2^{\mathrm{e}}$ période, le rapport $\frac{\mathrm{GMQ}_{2}-\mathrm{GMQ}_{1}}{\mathrm{GMQ} Q_{1}}$ traduit l'augmentation ou la réduction relative de l'intensité de croissance observée dans les lots BH ou HB après le changement de niveaux alimentaires.

La composition corporelle est déterminée par l'analyse chimique de ses constituants : eau, cendres, protéines, lipides. Les coefficients de rétention de l'azote et de l'énergie sont calculés selon des méthodes décrites dans les études antérieures (Desmoulin, 1967).

\section{RÉSULTATS}

Les résultats concernent la consommation et la croissance d'une part, la composition corporelle et l'efficacité des rétentions d'autre part. Ils sont rapportés successivement lorsque le niveau de rationnement est continu ( $\mathrm{HH}$ et $\mathrm{BB}$ ) puis lorsque 
celui-ci est variable (BH et $\mathrm{HB}$ ). $\mathrm{L}_{\mathrm{a}}$ figure 2 ci-dessous représente les courbes de croissance selon le type de rationnement et la durée des périodes.

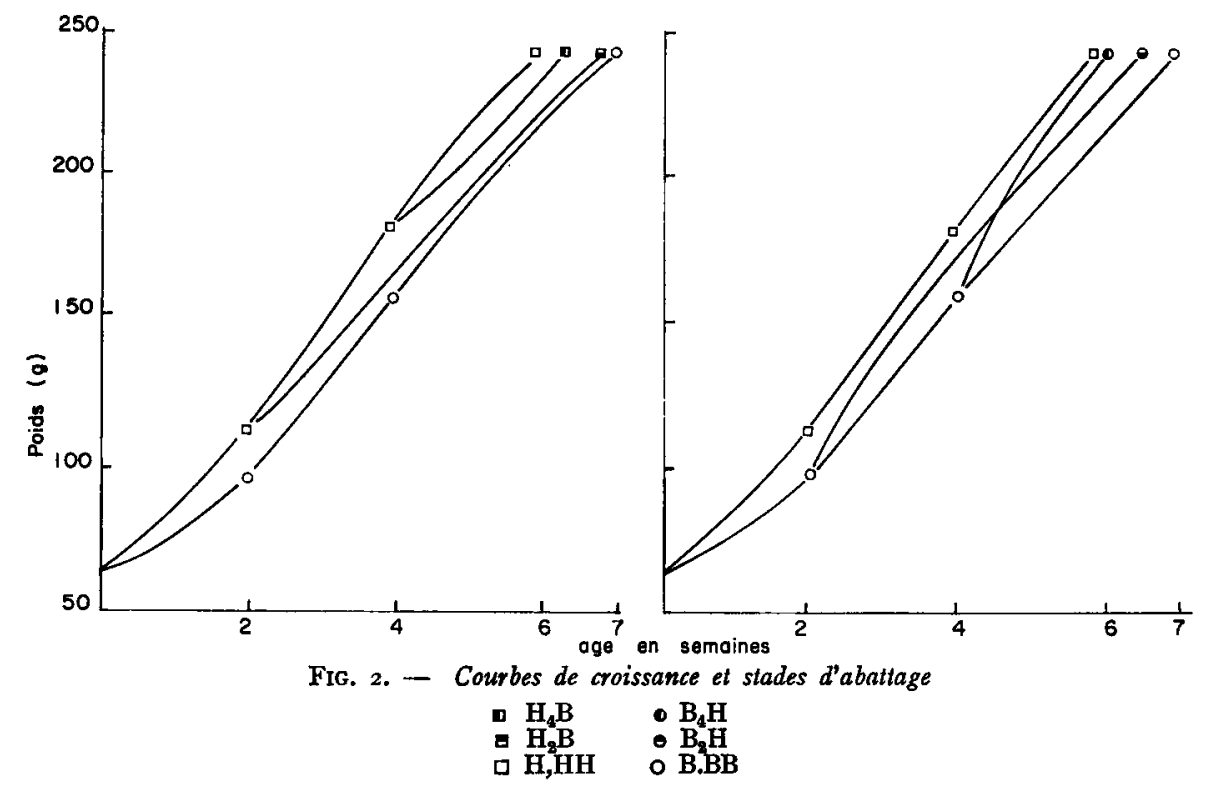

\section{A. - Le rationnement continu}

\section{Quantités consommées - Vitesse de croissance.}

- Pour une même quantité de protéines ingérée chez les animaux témoins $(\mathrm{H})$ et restreints (B), le tableau I montre que la réduction de $25 \mathrm{p}$. Ioo de l'apport énergétique réduit la vitesse de croissance de $30 \mathrm{p}$. I00 à 2 semaines, de $2 \mathrm{I} \mathrm{p}$. I00 à 4 semaines. Au poids final identique de $242 \mathrm{~g}(s x= \pm 3 \mathrm{~g})$ les animaux $\mathrm{BB}$ ont une croissance inférieure de $\mathrm{I} 5 \mathrm{p}$. Ioo à celle des témoins $\mathrm{HH}$; à ce stade, la consommation totale de protéines est accrue de 2I p. Ioo chez les animaux restreints, alors que la consommation totale d'énergie est comparable à celle des animaux témoins.

- Une réduction constante de l'apport énergétique a donc sur l'intensité de croissance des effets qui s'atténuent avec l'âge. $L_{a}$ vitesse de croissance maxima permise par le niveau d'alimentation est atteinte plus rapidement chez les animaux témoins (4 semaines) que chez les animaux restreints (8 semaines).

\section{Composition corporelle - Efficacité des rétentions.}

- Chez les animaux témoins et restreints, la composition corporelle est différente à 2 semaines, semblable à 4 semaines et au même poids final d'abattage : les protéines représentent un pourcentage à peu près constant du gain de poids, bien que cette proportion soit accrue de façon faible mais systématique chez les animaux restreints. Par contre, la teneur en lipides, fortement réduite à 2 semaines chez les animaux restreints, augmente ensuite chez ces derniers plus rapidement que chez les témoins. 
TABLEAU I

Consommation d'energie et de protéines

selon le niveau d'alimentation

et le stade d'abattage

\begin{tabular}{|c|c|c|c|c|c|c|}
\hline Stade d'abattage & \multicolumn{2}{|c|}{2 semaines } & \multicolumn{2}{|c|}{4 semaines } & \multicolumn{2}{|c|}{ Poids égal (1) } \\
\hline Lots & $\mathbf{H}_{\mathbf{2}}$ & $\mathbf{B}_{\mathbf{2}}$ & $\mathrm{H}_{4}$ & $\mathbf{B}_{\mathbf{4}}$ & HH & $\mathbf{B B}$ \\
\hline Durée des périodes (jours) ..... & 14 & 14 & 28 & 28 & 42 & 50 \\
\hline Poids final (1) (g) ........... & 112 & 97 & 182 & 158 & 242 & 242 \\
\hline $\begin{array}{c}\text { Quantité tot. } \\
\text { ingérée }\end{array}\left\{\begin{array}{l}\text { Énergie (kcal).. } \\
\text { Protéines (g)... }\end{array}\right.$ & $\begin{array}{l}710 \\
19,3\end{array}$ & $\begin{array}{l}544 \\
20,1\end{array}$ & $\begin{array}{r}1667 \\
45,3\end{array}$ & $\begin{array}{r}1274 \\
47,1\end{array}$ & $\begin{array}{r}2784 \\
75,6\end{array}$ & $\begin{array}{r}2652 \\
97,9\end{array}$ \\
\hline Croissance $(g / j) \ldots \ldots \ldots \ldots \ldots$ & 3,47 & 2,42 & 4,23 & 3,36 & 4,27 & 3,62 \\
\hline Croissance relative au témoin.... & 100 & 70 & 100 & 79 & 100 & 85 \\
\hline
\end{tabular}

(1) Écart-type $S \bar{x}= \pm 3 \mathrm{~g}$.

Dans le tableau 2, on peut noter à 2 semaines, une réduction de l'efficacité des rétentions azotées et énergétiques chez les animaux restreints. Ultérieurement, alors que l'efficacité azotée reste toujours plus faible chez les animaux restreints, l'efficacité énergétique devient égale à celle des animaux témoins. La restriction appliquée dès le sevrage, stimule ainsi le rendement de l'utilisation de l'énergie à la suite d'une adaptation progressive au niveau de rationnement bas.

TABLEAU 2

Composition corporelle (p. roo du poids frais) efficacité des rétentions (p. 100 des quantités ingérées)

\begin{tabular}{|c|c|c|c|c|c|c|c|}
\hline \multicolumn{2}{|c|}{ Stade de croissance } & \multicolumn{2}{|c|}{2 semaines } & \multicolumn{2}{|c|}{4 semaines } & \multicolumn{2}{|c|}{ Poids égal } \\
\hline & Lots & $\mathrm{H}_{2}$ & $\mathrm{~B}_{2}$ & $\mathrm{H}_{4}$ & $\mathrm{~B}_{4}$ & HH & $\mathrm{BB}$ \\
\hline $\begin{array}{c}\text { Composition } \\
\text { corporelle }\end{array}$ & $\left\{\begin{array}{l}\text { Eau .......... } \\
\text { Cendres ....... } \\
\text { Proténes ... . . } \\
\text { Lipides... . . . }\end{array}\right.$ & $\begin{array}{r}68,3 \\
3,2 \\
18,0 \\
10,5\end{array}$ & $\begin{array}{c}70,0^{*} \\
3,2 \\
18,8 \\
8,0^{*}\end{array}$ & $\begin{array}{r}64,9 \\
3,2 \\
18,0 \\
13,8\end{array}$ & $\begin{array}{r}65,9 \\
3,1 \\
18,9 \\
12,1\end{array}$ & $\begin{array}{r}63,0 \\
3,1 \\
18,6 \\
15,3\end{array}$ & $\begin{array}{r}63,3 \\
3,2 \\
19,3 \\
14,1\end{array}$ \\
\hline C.U.P. & $\left\{\begin{array}{l}\text { Azote } \ldots \ldots \ldots \\
\text { Energie } \ldots \ldots \ldots\end{array}\right.$ & $\begin{array}{l}42,4 \\
14,2\end{array}$ & $\begin{array}{l}32,6^{*} \\
10,5^{*}\end{array}$ & $\begin{array}{l}44,6 \\
17,7\end{array}$ & $\begin{array}{l}37,8^{*} \\
17,6\end{array}$ & $\begin{array}{l}43,5 \\
17,1\end{array}$ & $\begin{array}{l}35,6^{*} \\
17,4\end{array}$ \\
\hline
\end{tabular}

* Différence significative au seuil 0,05 . 


\section{B. - Le rationnement variable}

\section{Quantités consommées - Vitesse de croissance.}

- Pour un même gain de poids, les quantités totales consommées sont fonction du niveau d'alimentation et de la durée respective des périodes de rationnement Haut (H) et Bas (B). La consommation totale de protéines est ainsi accrue de $\mathrm{I} 8 \mathrm{p}$. Ioo dans le lot $\mathrm{H}_{2} \mathrm{~B}$ et la consommation totale d'énergie est inférieure de II $p$. roo dans le lot $\mathrm{B}_{4} \mathrm{H}$ relativement aux quantités consommées par ies animaux témoins $\mathrm{HH}$; les autres variations ne sont pas significatives.

- Relativement à la vitesse de croissance atteinte au terme de la première période de rationnement $\left(G M Q_{1}\right)$, la vitesse de croissance durant la seconde période $\left(\mathrm{GMQ}_{2}\right)$ traduit l'influence du changement de niveaux d'alimentation selon le stade de croissance ou la durée des périodes (tabl. 3).

a) Dans les lots $B H$, la vitesse de croissance augmente de 88 p. Ioo après 2 semaines de rationnement Bas, de 59 p. Ioo après 4 semaines de ce rationnement Bas. Toutefois, entre le stade 2 semaines et le stade 4 semaines, les animaux soumis au rationnement Bas-Bas ont augmenté de $38 \mathrm{p}$. Ioo leur propre vitesse de croissance. Ainsi, les animaux du lot $\mathrm{B}_{4} \mathrm{H}$ récupèrent finalement leur retard de croissance, plus rapidement que ceux du lot $\mathrm{B}_{2} \mathrm{H}$.

TABLEAU 3

Consommation d'énergie et de protéines selon la durée des périodes $\grave{a}$ poids égal d'abattage : $242 \mathrm{~g}(s \bar{x}=+3 \mathrm{~g})$

\begin{tabular}{|c|c|c|c|c|}
\hline Changement de niveaux & \multicolumn{2}{|c|}{2 semaines } & \multicolumn{2}{|c|}{4 semaines } \\
\hline Lots & $\mathrm{H}_{2} \mathrm{~B}$ & $\mathrm{~B}_{2} \mathrm{H}$ & $\mathrm{H}_{4} \mathrm{~B}$ & $\mathrm{~B}_{4} \mathrm{H}$ \\
\hline Durée de l'expérience $(j) \ldots \ldots \ldots$ & 49 & 46 & 45 & 43 \\
\hline $\begin{array}{c}\text { Quantité tot. } \\
\text { ingérée }\end{array}\left\{\begin{array}{l}\text { Énergie (kcal) } \ldots \\
\text { Protéines }(\mathrm{g}) \ldots .\end{array}\right.$ & $\begin{array}{l}2617 \\
\quad 89,7\end{array}$ & $\begin{array}{l}2792 \\
81,2\end{array}$ & $\begin{array}{l}2749 \\
\quad 85,1\end{array}$ & $\begin{array}{l}2500 \\
80,4\end{array}$ \\
\hline $\begin{array}{c}\text { Croissance } \\
(\mathrm{g} / \mathrm{j})\end{array}\left\{\begin{array}{l}1^{\text {re }} \text { période } \\
2^{\mathrm{e}} \text { période.................... }\end{array}\right.$ & $\begin{array}{l}3,50 \\
3,77\end{array}$ & $\begin{array}{l}2,40 \\
4,52\end{array}$ & $\begin{array}{l}4,27 \\
3,49\end{array}$ & $\begin{array}{l}3,44 \\
5,48\end{array}$ \\
\hline Total périodes ... & 3,69 & 3,88 & 3,97 & 4,15 \\
\hline Variation relative de croissance (1). & +8 & +88 & -18 & +59 \\
\hline
\end{tabular}

(1) Croissance (2e période-1 ${ }^{\mathrm{re}}$ période)/croissance $1^{\mathrm{r} \theta}$ période.

b) Dans les lots $H B$, la vitesse de croissance n'est pas inférieure à celle observée après 2 semaines de rationnement Haut. Par contre, après 4 semaines, elle est réduite de I8 p. Ioo. Or, les animaux soumis au rationnement Haut-Haut ont augmenté de 
2 I p. Ioo leur vitesse de croissance entre le stade 2 semaines et le stade 4 semaines. Aussi les animaux du lot $\mathrm{H}_{4} \mathrm{~B}$ conservent au même poids final un avantage de croissance sur ceux du lot $\mathrm{H}_{2} \mathrm{~B}$.

En définitive, dans les lots $\mathrm{H}_{2} \mathrm{~B}$ et $\mathrm{BB}$ seuls, la vitesse de croissance est plus faible que celle observée dans le lot témoin $\mathrm{HH}$.

Composition corporelle - Efficacité des rétentions.

A poids égal d'abattage, la composition corporelle rapportée dans le tableau 4, est fonction du type de rationnement adopté : Bas-Haut (BH) ou Haut-Bas (HB). L'effet de l'inversion des niveaux de rationnement est présenté dans le tableau 5 .

TABL,EAU 4

Composition corporelle ( $\mathrm{p}$. roo du poids frais) Efficacité des rétentions (p. Ioo des quantités ingérées)

\begin{tabular}{|c|c|c|c|c|c|}
\hline \multicolumn{2}{|c|}{ Lots } & $\mathrm{H}_{2} \mathrm{~B}$ & $\mathrm{~B}_{2} \mathrm{H}$ & $\mathrm{H}_{4} \mathrm{~B}$ & $\mathrm{~B}_{4} \mathrm{H}$ \\
\hline $\begin{array}{l}\text { Composition } \\
\text { corporelle }\end{array}$ & 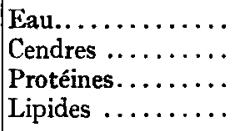 & $\begin{array}{c}63,6 \\
3,3 \\
19,7 \\
13,4^{*}\end{array}$ & $\begin{array}{r}62,4 \\
3,2 \\
18,7 \\
15,7\end{array}$ & $\begin{array}{c}64,2 \\
3,2 \\
19,3 \\
13,2^{*}\end{array}$ & $\begin{array}{r}61,8 \\
3,2 \\
18,4 \\
16,6\end{array}$ \\
\hline C.U.P. & $\begin{array}{l}\text { Azote } \ldots \ldots \ldots \ldots \\
\text { Énergie } \ldots \ldots \ldots\end{array}$ & $\begin{array}{l}39,7 \\
17,1\end{array}$ & $\begin{array}{l}40,5 \\
17,4\end{array}$ & $\begin{array}{l}42,1 \\
16,5\end{array}$ & $\begin{array}{l}40,2 \\
20,1^{*}\end{array}$ \\
\hline
\end{tabular}

* Différence significative au seuil 0,05 .

TABLEAU 5

Gain en protéines et en lipides (p. Ioo du gain de poids) après le changement de niveau d'alimentation

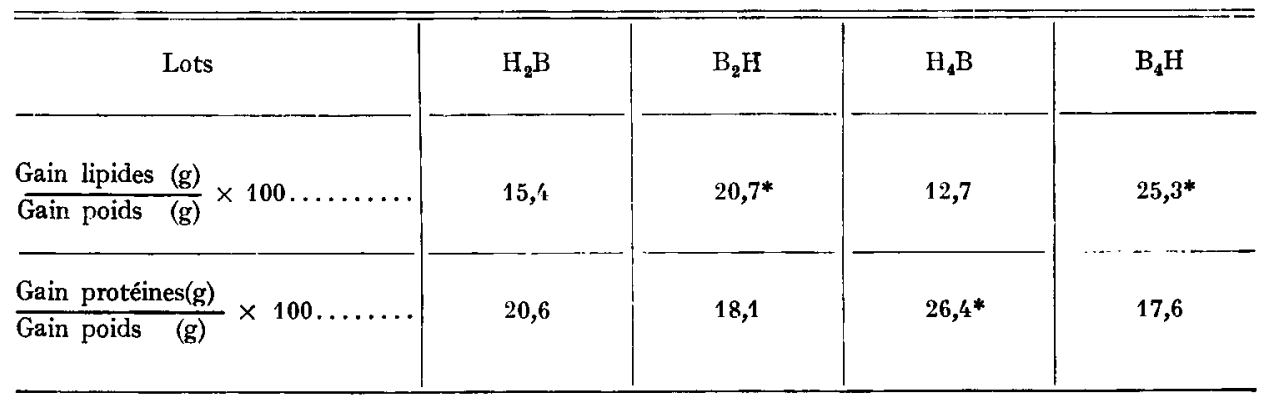

* Différence significative au seuil 0,05 . 
Quel que soit le stade de croissance, la teneur en lipides du gain de poids est accrue dans les lots BH et réduite dans les lots HB. On remarque chez les animaux du lot HB une augmentation de la teneur en protéines du gain de poids après le changement de niveaux à 4 semaines.

La comparaison et l'évolution des constituants énergétiques corporels suivant le type de rationnement apparaissent sur la figure 3 .

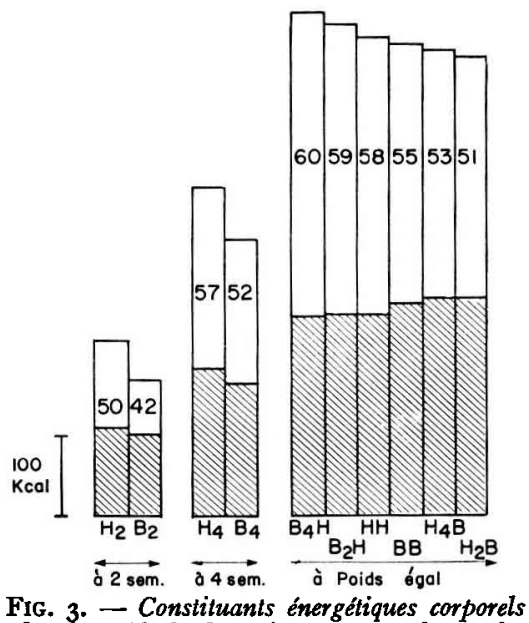

Évolution selon la methode de rationnement et les stades d'abattage

$$
\begin{aligned}
& \text { calories protéiques } \\
& \text { calories lipidiques } \\
& \text { P. roo calories lipidiques } \\
& \text { calories totales }
\end{aligned}
$$

L'augmentation des calories lipidiques en pourcentage des constituants énergétiques corporels (protéines et lipides) caractérise l'évolution de la lipogenèse. A 2-4-6 semaines, 1'évolution 42-52-55 dans le lot restreint est comparée aux valeurs 50-57-58 dans le lot témoin. La lipogenèse, précoce dans le lot $\mathrm{HH}$, est tardive dans le lot BB restreint de façon continue depuis le sevrage. Dans les lots $\mathrm{BH}, 1$ 'augmentation de la lipogenèse est représentée par l'évolution 42-59 lors du changement à 2 semaines et par l'évolution 52-60 lors du changement à 4 semaines. Dans les lots HB l'évolution 50-5 I lors du changement à 2 semaines traduit le maintien de la lipogenèse à un niveau faible. Par contre, lors du changement à 4 semaines, l'évolution 57-53 indique une réduction de la lipogenèse à ce stade.

Au même poids final, les différences observées relativement aux animaux du lot témoin HH sont les suivantes : pour les lots HB seuls, l'adiposité est réduite. Par contre, dans le lot $\mathrm{BB}, 1$ 'adiposité reste comparable à celle observée dans le lot témoin.

Suivant le stade auquel s'applique la restriction alimentaire, l'efficacité des rétentions énergétiques et azotées subit les modifications suivantes : l'efficacité azotée est abaissée chez les animaux soumis plus longtemps au rationnement Bas (lots $\mathrm{BB}-\mathrm{B}_{4} \mathrm{H}$ et $\mathrm{H}_{2} \mathrm{~B}$ ). L'efficacité énergétique est croissante dans les lots $\mathrm{BH}$ et décroissante dans les lots $\mathrm{HB}$ lorsque la $\mathrm{I}^{\mathrm{re}}$ période de rationnement est plus longue. 


\section{DISCUSSION}

\section{Le rationnement continu}

Les résultats de cette expérience montrent que les animaux restreints de $25 \mathrm{p}$. Ioo en énergie depuis le sevrage, conservent la possibilité d'augmenter leur vitesse de croissance à un âge auquel les animaux témoins ont exprimé la majeure partie des possibilités de croissance permises par un niveau élevé d'alimentation. Ces résultats sont en accord avec ceux de DuRAND et al. ( 1967 ) qui montrent que les animaux restreints conservent un pouvoir de multiplication cellulaire plus élevé que les animaux témoins de même âge. Le maintien des animaux à un stade juvénile consécutif à un retard de croissance a été également mis en évidence par MCROBERTs (I965), McINTYRE et Gardiner (I964) chez les Oiseaux. Holeckova (I964) note de plus chez le Rat les effets favorables d'un jeûne intermittent sur l'âge biologique de l'animal. Enfin, Brody (I945), MoNTEIro et FALCONER (I966) rapportent que le niveau de nutrition modifie 1'âge mais non le poids auquel serait atteinte la maturité sexuelle.

Chez l'animal restreint, l'augmentation tardive des dépôts lipidiques dans le gain de poids peut en outre résulter de la composition des régimes de sevrage et des conséquences du rationnement Bas sur le comportement alimentaire. Plusieurs auteurs, TEPPERMAN (I958-Ig6r), HegGeness (I96r), FABRy (I962), JACQUOT et al. (I966), notent en effet chez le rat adapté à une alimentation discontinue, que la consommation rapide des régimes riches en glucides provoque une stimulation de la lipogenèse. Enn réduisant l'apport alimentaire, les animaux affamés consomment rapidement leurs repas : ceci peut donc entraîner progressivement 1'évolution décrite ci-dessus. Soulignons que le sevrage, phase critique de la vie de l'animal, est une période d'orientation de l'activité des systèmes enzymatiques en raison du changement des conditions nutritionnelles (TAYLOR et al., I967).

\section{Le rationnement variable}

Une variation du niveau de rationnement au cours de la croissance entraine des modifications de croissance et de composition corporelle en accord avec les résultats observés en rationnement continu.

a) Dans les lots $B H$, l'intensité de la récupération ou de croissance compensatrice diminue avec l'âge lorsque le rationnement Bas est plus prolongé. Toutefois, en valeur relative, le retard de croissance est plus faible à 4 semaines qu'à 2 semaines. Il en résulte que l'animal réalimenté plus tardivement peut rattraper plus vite ce retard. Pour la durée des périodes de restriction alimentaire, considérée après le sevrage, ces résultats sont conformes à ceux obtenus par MEYER et al. (I956) et WIDDOWson (I964). En fonction de la durée de la sous-nutrition, la lipogenèse est plus fortement stimulée après 4 semaines. En alimentation discontinue (MORIN-JoMAIN et JACQUOT, I967) cette stimulation serait toutefois moins forte lorsque les régimes sont hyperlipidiques. 
A la suite d'une restriction alimentaire appliquée dès le seurage, une réalimentation même contrôlée entraîne une augmentation de la lipogenèse; par contre, elle permet d'augmenter l'efficacité nutritionnelle.

b) Dans les lots $H B$, la vitesse de croissance est réduite à la suite d'un rationnement Haut, prolongé durant 4 semaines. Par contre, elle se maintient à un niveau faible lorsque le rationnement Bas a été imposé 2 semaines après le début de l'expérience. La lipogenèse suit une évolution parallèle à celle de la vitesse de croissance. Par ailleurs, une augmentation de la concentration en protéines du gain de poids est observée après 4 semaines de rationnement Haut ; ceci est conforme aux résultats de DURAND et al (I967), selon lesquels la carence en énergie a des effets modérés sur la synthèse des protéines. Cependant, la protéinogenèse s'effectue avec un rendement d'autant plus faible que la restriction en énergie s'applique tôt après le sevrage.

Contrairement à l'évolution de la composition corporelle observée dans le lot BB, la lipogenèse n'est pas stimulée dans le lot $\mathrm{HB}$, lorsque la restriction alimentaire est appliquée à la suite d'un niveau élevé d'alimentation au sevrage. Dans nos conditions expérimentales, le rationnement Haut conduit à une lipogenèse maxima vers 1'âge de 7 semaines. La sévérité de la restriction à imposer est fonction du stade de croissance auquel elle est appliquée (DESMOULIN, I967). Les travaux de TAYLOR et al. (I967) montrent ainsi que l'activité des enzymes favorisant la lipogenèse hépatique présente un maxima 2 à 3 semaines après le sevrage en alimentation à volonté. Il semble donc important de tenir compte de cette évolution de la lipogenèse selon l'âge pour déterminer l'intensité et la durée de la restriction alimentaire qui permettra d'obtenir un animal maigre à un poids donné d'abattage.

Le maintien d'un niveau élevé d'alimentation après le sevrage apparaît nécessaire pour qu'une restriction alimentaire appliquée ultérieurement, permette de limiter la lipogenèse. Toutefois, ce résultat est obtenu au détriment de l'efficacité nutritionnelle.

Reçu pour publication en mars 1968.

\section{SUMMARY}

\section{INFLUENCE OF DURATION OF PERIODS OF RESTRICTED INTAKE OF FEED AFTER WEANING} ON DEVELOPMENT OF BODY COMPOSITION IN WHITE RATS

An experiment was done on Wistar white rats to study the influence of the plane of nutrition and the length of time it was imposed after weaning on the later development of body composition. For this, the control and restricted rats were given the same amount of protein daily but supplies of energy which differed quantitatively by 25 per cent. The two planes of nutrition, high $(\mathrm{H})$ and low (B) were interchanged either 2 or 4 weeks after the start of the experiment. In the groups given varied rationing, low-high $(\mathrm{BH})$ and high-low $(\mathrm{HB})$, the results for body composition were compared with those obtained in the $\mathrm{HH}$ and $\mathrm{BB}$ groups, which had been kept on a constant ration.

Continuous restriction of supply of energy from weaning dit not reduce growth rate in a constant way. It was reduced by 30 per cent at 2 weeks, by 21 per cent at 4 weeks and by 15 per cent when the rats were killed at a wight equal to that of controls. The restricted animals had smaller lipid deposits only at the start of growth. After this the deposits increased slowly so that at equal weight the controls $(\mathrm{HH})$ and restricted rats $(\mathrm{BB})$ had similar body composition. The development of lipogenesis was the result of adaptation of the animals to the plane of nutrition imposed on the one hand and the effect of restriction on the feeding behaviour of the animals which were given a limited amount of a diet rich in carbohydrates, on the other.

After the change in the plane of nutrition development of body composition depended essentially on the plane imposed at weaning and the length of time that it was imposed after weaning. The development was characterized mainly by changes in lipogenesis : in the $\mathrm{BH}$ groups it increased 
more when the time on the restricted intake was longer. The high plane clearly demonstrated the adaptation of the restricted animals to limitation of their energy expenditure. Efficiency of feed utilization increased in the same way as fat deposit.

In the HB groups the effect of restriction of feed on the development of lipogenesis depended on the stage of development of the animal. At 2 weeks lipogenesis remained at a low level ; at 4 weeks it was reduced. In each case fat deposit was less than that seen in control animals of the same weight, but efficiency of feed utilization was reduced.

The severity of restriction to be imposed according to the stage of growth must be defined in looking for criteria by which the aptitude of the animals for lipid deposition during the critical phases of growth after weaning may be characterized.

\section{RÉFÉRENCES BIBLIOGRAPHIQUES}

BRoDY S., 1945. Bioenergetics and growth. New York ReinHold.

Clarke M. F., Smith A. H., 1938. Recovery following suppression of growth in the rat. Brit. J. Nuir., $15,245^{-25}$.

Desmoulin B., 1967. Évolution selon l'âge et le poids de la composition corporelle du rat blanc soumis à un rationnement énergétique après le sevrage. Ann. Biol. anim. Bioch. Biophys., 7, 281-293.

Desmoulin B., 1967. Note sur l'influence de la sévérité du rationnement énergétique sur la composition corporelle du rat blanc après le sevrage. Ann. Biol. anim. Bioch. Biophys., 7, 295-301.

Durand G., Fauconneau G., Éliane Penot., 1967. Croissance des tissus du Rat et réduction de l'apport

énergétique de la ration. Influence sur la teneur en acides nucléiques. Ann. Biol. anim. Bioch. Biophys., 7, I45-155.

Fabry P., Petrasek R., Braun T., Bednarek M., Horakova E., Konopasek E., 1962. Lipogenesis in rats adapted to intermittent starvation or continuous underfeeding. Experentia, 18, 555-558.

FABRY P., 1964. Der einfluss der frequenz der Nahrungsaufnahme auf den Fettstoffwechsel. Die Nahrung., 8, 304-310.

HEGGENESS F. W., I96r. Metabolic Rate and lipogenesis in weanling rats fed High carbohydrate diets. Am. J. Physiol., 200, 80-84.

HEGGENESS F. W., 1965. Effect of intermittent food restriction on growth, food utilization and body composition of the rat. J. Nutr., 88, 265-270.

Holeckova E., 1964. Influence du jeûne sur l'âge biologique chez le Rat. J. Physiol., 56, 570-571.

JACQUOT R., Morin-JomaiN M., ABRAHAM J., I966. Influence de l'alimentation protéique discontinue sur le comportement nutritionnel du rat blanc. Amino Acides peptides proteines. Cahier n $n^{\circ}$ AEC., 195-298.

Mc Cay C. N., Maynard L. A., Sperling G., Barnes L. L., 1939. Retarded growth life-span, ultimate body size and age changes in the albino rats after feeding diets restricted in calories. J. Nutr., 18, I-I4.

MC INTYRE T. M., GARDINER E. E., 1964. Effect of duration of feed restriction on the laying house performance of confinement reared pullets. Poultry. Sci., 43, 467-473.

MC MEEKAN C. P., I940. Growth and development in the pig with special reference to carcass_quality characteristics. J. Agric. Sci., 30, 276-569.

MCROBERTs M., 1965. Growth retardation of day-old chickens and physiological effects at maturity. $J$. Nutr., 87, 3I-40.

MeYer J. H., Luecker C. E., SMith J. D., 1956. Influence of food and energy restriction and subsequent recovery on body composition and food utilisation of rats. J. Nutr., 60, I21-r28.

Monteiro L. S., FalConer D. S., 1966. Compensatory growth and sexual maturity in mice. Anim. Prod., 8, i79-192.

MoRIN-JomaIN M., I962. Le comportement nutritionnel du rat soumis à des restrictions alimentaires périodiques suivies de réalimentation. Arch. Sci. Physiol., 16, 441-456.

MoRIN-JoMaIN M., I963. Le comportement nutritionnel du rat soumis alternativement à des régimes protéiprives et à des régimes protéiques. Arch. Sci. Physiol., 17, 55-74.

MORIN-JomaIN M., JACQUOT R., 1967. Comportement alimentaire. Rythme alimentaire, croissance et composition corporelle chez le Rat. Cah. Nutr. Diét., 2, 43-49.

OsBorne T. B., MENDEL L. B., 1915. The resumption of growth after long continued failure of growth. J. Biol. Chem., 23, 439-454.

TAYlOR C. B., Bailey E., Bartley W., 1967. Changes in hepatic lipogenesis during development of the rat. Biochem. J. 105, 717-722.

Tepperman H. M., Tepperman J., 1961. Metabolism of glucose ${ }^{-}-C^{14}$ and glucose 6-C $\mathrm{C}^{14}$ by liver slices of refed rats. Amer. J. Physiol., 200, 1069-1073.

WiLson P. N., Osbourn D. F., 1960. Compensatory growth after undernutrition in mammals and birds. Biol. Reviews., 35, 324-363.

WidDowson E. M., 1964. Early nutrition and later development. Diet and Bodily constitution. Ciba foundation A. Churchill. London 3-18. 